\title{
Corrections and enhancements of quasi-equilibrium states
}

\author{
Alexander N. Gorban ${ }^{\text {a }}$, Iliya V. Karlin ${ }^{\text {b,* }}$, Patrick Ilg ${ }^{b}$, \\ Hans Christian Öttinger ${ }^{b}$ \\ a Institute of Computational Modeling RAS, 660036 Krasnoyarsk, Russia \\ ${ }^{\mathrm{b}}$ ETH Zürich, Department of Materials, Institute of \\ Polymers, CH-8092 Zürich, Switzerland
}

Received 4 October 1999; accepted 7 February 2000

\begin{abstract}
We give a compact non-technical presentation of two basic principles for reducing the description of nonequilibrium systems based on the quasi-equilibrium approximation. These two principles are: construction of invariant manifolds for the dissipative microscopic dynamics, and coarse-graining for the entropy-conserving microscopic dynamics. Two new results are presented: first, an application of the invariance principle to hybridization of micro-macro integration schemes is introduced, and is illustrated with non-linear dumbbell models; second, Ehrenfest's coarse-graining is extended to general quasi-equilibrium approximations, which gives the simplest way to derive dissipative equations from the Liouville equation in the short memory approximation. (C) 2001 Elsevier Science B.V. All rights reserved.
\end{abstract}

Keywords: Non-equilibrium thermodynamics; Quasi-equilibrium approximation; Invariant manifold; Coarse-graining

\section{Quasi-equilibrium approximations and their place in the problem of transition from microscopic to macroscopic variables}

Most of the works on non-equilibrium thermodynamics deal with corrections to quasi-equilibrium approximations or with applications of these approximations (with or without corrections). This viewpoint is not only possible but it proves very efficient for the construction of a variety of useful models, approximations and equations, as well as methods to solve them.

\footnotetext{
* Corresponding author. Fax: +41-1-632-10-76.

E-mail address: ikarlin@ifp.mat.ethz.ch (I.V. Karlin).

0377-0257/01/\$ - see front matter @ 2001 Elsevier Science B.V. All rights reserved.

PII: S0377-0257(00)00135-X
} 
The quasi-equilibrium approximation is based on the entropy maximum principle under fixed constraints [1-8]: ${ }^{1}$

$$
S(f) \rightarrow \max , \quad m(f)=M,
$$

where $f$ is the microscopic state, $S$ is the entropy, and $M$ are the values of the macroscopic variables of a reduced description. Operators $m$ map $f$ into $M$. In most cases, operators $m$ are linear, while $S$ is concave, and then if the solution to the problem (1) exists, it is unique. The solution to Eq. (1) is called the quasi-equilibrium state, and it will be denoted as $f^{*}(M) .{ }^{2}$ The classical example is the local equilibrium of a simple gas: $f$ is the one-body distribution function, $S$ is the Boltzmann entropy, $m$ are five linear operators, $m(f)=\int\left\{1, \boldsymbol{v}, v^{2}\right\} f \mathrm{~d} \boldsymbol{v}$, with $\boldsymbol{v}$ being the particle's velocity; corresponding $f^{*}(M)$ is called the local Maxwell distribution function. Further examples will be encountered later on. Several branches of modern non-equilibrium thermodynamics are built upon the quasi-equilibrium approximation, most notably and explicitly, the extended irreversible thermodynamics [9].

When the macroscopic variables $M$ span the range of their admissible values, the quasi-equilibrium state spans a set of states, the quasi-equilibrium manifold $\Omega^{*}$. It is useful to visualize the quasi-equilibrium manifold (see Fig. 1).

If the microscopic dynamics is given by an equation

$$
\dot{f}=J(f),
$$

then the quasi-equilibrium dynamics of the variables $M$ reads

$$
\dot{M}=\left.\langle\operatorname{Dm}(f) \mid J(f)\rangle\right|_{f^{*}(M)} .
$$

Here, $\operatorname{Dm}(f)$ is the differential of $m$ - linear operator, and we use shorthand notation $\langle g \mid h\rangle$ for the scalar product between the elements of the phase space of Eq. (2).

The quasi-equilibrium approximation has an important property, it conserves the type of the dynamics: if the entropy increases monotonically (or not decreases) due to Eq. (2), then the same is true for the quasi-equilibrium entropy $S^{*}(M)=S\left(f^{*}(M)\right)$ due to the quasi-equilibrium dynamics (3). That is, if

$$
\dot{S}=\langle D S(f) \mid \dot{f}\rangle=\langle D S(f) \mid J(f)\rangle \geq 0,
$$

then

$$
\dot{S}^{*}=\sum_{k} \frac{\partial S^{*}}{\partial M_{k}} \dot{M}_{k}=\left.\sum_{k} \frac{\partial S^{*}}{\partial M_{k}}\left\langle D m_{k}(f) \mid J(f)\right\rangle\right|_{f^{*}(M)}=\left.\langle D S(f) \mid J(f)\rangle\right|_{f^{*}(m)} \geq 0 .
$$

\footnotetext{
${ }^{1}$ From time to time, it is discussed in the literature as to who was the first to introduce the quasi-equilibrium approximations, and how to interpret them. At least a part of the discussion is due to a different role the quasi-equilibrium plays in the entropy-conserving and the dissipative dynamics. The very first use of the entropy maximization dates back to the classical work of G.W. Gibbs [1], but it was first claimed for a principle by E.T. Jaynes [2]. Probably, the first explicit and systematic use of quasi-equilibria to derive dissipation from entropy-conserving systems is due to the works of D.N. Zubarev (recent detailed exposition is given in [3]). For dissipative systems, the use of the quasi-equilibrium to reduce description can be traced to the works of H. Grad on the Boltzmann equation [4]. The viewpoint of two of the present authors (ANG and IVK) was influenced by the papers by L.I. Rozonoer and co-workers, in particular A.M. Kogan and L.I. Rozonoer [5-7]. A detailed exposition of the quasi-equilibrium approximation for Markov chains is given in [8].

${ }^{2}$ In this paper, the notion of quasi-equilibrium refers solely to the context of problem (1), and 'quasi' does not obligatorily imply 'near'. Alternatively, $f^{*}(M)$ is sometimes called the 'generalized canonical state' or simply the 'maximum entropy state'.
} 


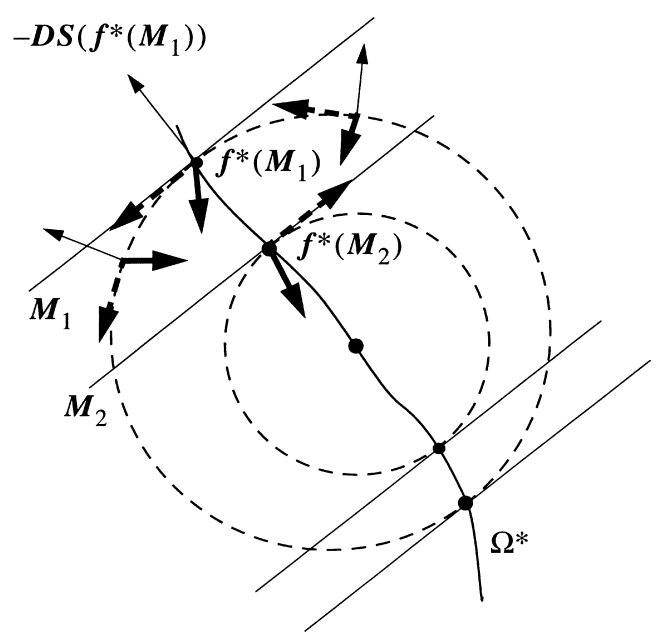

Fig. 1. The quasi-equilibrium manifold $\Omega^{*}$ : dashed convex curves represent the levels of the entropy. Each straight solid line represents the plane which contains all states with the fixed values of macroscopic parameters. The values of macroscopic parameters are different on different planes. In the case of linear macroscopic parameters, the bunch of hyperplanes consists of parallel individual planes. The point where the plane touches the level of the entropy is the quasi-equilibrium state. The totality of these tangency points makes up the bold curve in the phase space called the quasi-equilibrium manifold $\Omega^{*}$. Dashed bold arrows, touching the levels of the entropy, represent the microscopic vector field in the case of the entropy-conserving dynamics. Solid bold arrows represent the microscopic vector field in the case of the dissipative dynamics. In the former case, the angle between the gradient of the entropy (thin arrows) is towards the right-hand side, in the latter case it is acute. Zero projection of dashed arrows onto the quasi-equilibrium manifold is the peculiarity of the two-dimensional picture.

Here, $D S(f)$ is the differential of $S-$ the linear functional, and summation in $k$ will always imply summation over the set of the macroscopic variables. The last line in Eq. (4) is the immediate implication of the identity

$$
\left.\sum_{k} \frac{\partial S^{*}}{\partial M_{k}} D m_{k}(f)\right|_{f^{*}(M)}=\left.D S(f)\right|_{f^{*}(M)},
$$

which follows from solving the problem (1) by the method of Lagrange multipliers, and that of the chain rule of differentiation.

Conservation of the type of dynamics by the quasi-equilibrium approximation is a simple yet a general and useful fact. If the entropy $S$ is an integral of motion of Eq. (2), then $S^{*}(M)$ is the integral of motion for the quasi-equilibrium equation (3). Consequently, if we start with a system that conserves the entropy (for example, with the Liouville equation), then we end up with the quasi-equilibrium system which conserves the quasi-equilibrium entropy. For instance, if $M$ is the one-body distribution function, and (2) is the (reversible) Liouville equation, then (3) is the Vlasov equation which is as well reversible, that is, the entropy does not change for it. On the other hand, if the entropy increases monotonically on solutions to Eq. (2), then the quasi-equilibrium entropy also increases monotonically on solutions to the quasi-equilibrium dynamic equations (3). For instance, if Eq. (2) is the Boltzmann equation for the one-body distribution function, and $M$ is a finite set of moments (chosen in such a way that the solution to the problem (1) exists), then (3) are closed moment equations for $M$ which increase the quasi-equilibrium entropy (this is the essence of a well-known generalization of Grad's moment method). 
Quasi-equilibrium approximations may be regarded as insufficient for various reasons such as inaccuracy, lack of a correct (in one's opinion) dissipation etc. How can one improve the quasi-equilibrium approximation? Practically, all routes of improvements can be grouped in two directions.

The first route comprises corrections - construction of a manifold which corresponds to an intuitive idea of time separation: solutions 'rapidly' approach this manifold and after that they 'slowly' move along this manifold. The absolute success in this direction means that we have constructed a stable invariant manifold. Various approximate methods follow this route. They may use small parameter expansions (for example, the Chapman-Enskog method of the theory of the Boltzmann equation which seeks corrections to the simplest quasi-equilibrium manifold, the local equilibrium), or instead, iteration methods of the Newton type. Along these lines, the quasi-equilibrium approximations are seen as natural initial approximations to the invariant manifolds. In Section 2, we outline the route of corrections as applied to dissipative systems, focusing on the invariance principle as the guide for corrections. In particular, we describe a novel application of the invariance principle in combining micro and macro computations, and demonstrate how this works for non-linear dumbbell models of non-Newtonian fluids.

But seeking invariant manifolds does not always lead to the desired goal. For example, following the route of corrections, it is impossible to derive dissipative systems from the Liouville equation because the entropy is conserved on any solution, and thus, no monotonic dissipation can be introduced. Therefore, a second route (which we call enhancement) is considered in Section 3. This is a formalization and extension of Ehrenfest's coarse-graining procedure [10], and it is aimed at deriving short-memory dissipative models from entropy-conserving dynamics. We prove the explicit entropy production formula for these models, and discuss the advantages and limitations of this construction. We close this introductory section with three comments.

1. Division into 'micro' and 'macro' is context-dependent: what is regarded as 'micro' in one statement of the problem can become 'macro' in a different context. For example, the one-body distribution function was the macroscopic variable in the first example mentioned above (derivation of kinetic equation from the Liouville equation), while it was the microscopic variable in the second example (derivation of moment equations from the kinetic equation). In a similar way, the dumbbell configuration function is the macroscopic variable when derived in a full-chain setting, while it is the microscopic variable when constitutive equations for the stress tensor are derived from dumbbell models, and so on.

2. In view of many possible paths from micro to macro, any reasonable procedure of coarse-graining the evolution equation should satisfy the following requirement of commutativity of diagrams:

$$
\Pi_{2 \rightarrow 3^{*}} \Pi_{1 \rightarrow 2}=\Pi_{1 \rightarrow 3} .
$$

Here, 1, 2, and 3 are different levels of description, and $\Pi_{i \rightarrow j}$ transforms from a microscopic level of description to a more macroscopic level. The diagram must be commutative: it should not matter as to which route of coarse-graining was taken to come from microscopic level 1 to macroscopic level 3 . One route $(1 \rightarrow 2 \rightarrow 3)$ goes stepwise, first to some intermediate level 2 and then from level 2 to level 3 . Another route is directly $1 \rightarrow 3$. The result (the macroscopic kinetic equations on level 3 ) is defined only by equations on level 1 , by the initial and the final choice of variables and cannot depend on the route given in diagram (5).

3. The connection between the entropy chosen to construct quasi-equilibrium approximations and the classical definition $-\int f \ln f \mathrm{~d} x-$ is rather conventional. In the first place, it is important that $S$ is an increasing (or not decreasing) Lyapunov function of the 'microscopic' dissipative system or the 
integral of motion if the system is entropy-conserving. Let us remind that Markov processes always have a whole family of non-decreasing Lyapunov functions:

$$
S_{h}=-\int f^{\mathrm{eq}}(x) h\left(\frac{f(x)}{f^{\mathrm{eq}}(x)}\right) \mathrm{d} x,
$$

where $f^{\text {eq }}$ is the equilibrium of the Markov process, and $h(y)$ is a strictly convex function of one variable, $h^{\prime \prime}(y)>0$.

If $h(y)=y \ln y$, then $S_{h}(6)$ is the usual entropy. The condition of additivity of the entropy under joining of statistically independent subsystems is satisfied by the one-parametric set of convex functions

$$
h_{\mathrm{ad}}(y)=\alpha y \ln y-(1-\alpha) \ln y,
$$

where $\alpha \in[0,1]$. The usual entropy is distinguished in this one-parametric set by continuity as $y \rightarrow 0$.

For computations, it is sometimes useful to consider the 'entropy' defined by $h(f)=f^{\alpha}$, where $\alpha>1$. It may be considered as an approximation to the additive entropy (7), but one also can seek separate physical motivations for that [11]. It is not the goal of this paper to discuss this point in any detail though motivations like multifractality, presence of long-range interactions or Lévy flights should be mentioned. From the perspective of methods which are seeking improvements on the quasi-equilibrium approximations, the choice of the entropy from the family (6) is, to a large extent, a matter of convention. If the quasi-equilibrium is considered only as an initial approximation, and not as the 'ultimate truth', then the requirements of the choice are different - they are dictated by convenience of computations, may be, by convergence reasoning and so on. In other words, if the quasi-equilibrium is introduced in a process of successive approximations, then the requirements of the choice of the entropy become technical rather having a fundamental character. Even the requirement of additivity can be violated 'on the way' to the final answer, and instead of (7) we, in principle, can take non-additive entropies. This will correspond to a violation of additivity and an effective many-body interaction. Yet another restriction on the choice of the entropy comes from equilibrium thermodynamics: if a problem of reduced description under consideration allows for matching it to the equilibrium thermodynamics in some limit, then the choice of the entropy should be consistent with the expected properties of the thermodynamic entropy in that limit.

Finally, one should keep in mind that there are dissipative systems where the question of the choice of the entropy does not appear. These are classical kinetic equations like the Boltzmann and Landau equations which have only one universal (that is, independent of the details of the interaction) Lyapunov function.

\section{The invariance principle}

\subsection{The method of invariant manifold for dissipative systems}

The method of invariant manifold $[12,13]$ is aimed at constructing the invariant manifold of slow motions $\Omega=f(M)$, which is parameterized with the values of the chosen macroscopic parameters $M$, that is, $m(f(M))=M$. Invariance means that the true vector field $J$ of Eq. (2) touches the manifold $\Omega$ in each of its points. If $f(t)$ is a solution to Eq. (2) with $f(0) \in \Omega$, then $f(t) \in \Omega$ for all times $t>0$, that is, solutions do not leave $\Omega$. 'Slowness' of the manifold $\Omega$ means that the time hierarchy hypothesis is 
applicable: solutions of Eq. (2) 'rapidly' approach $\Omega$, and after that move 'slowly' along it. It is natural to assume that such a behavior applies to solutions with the initial conditions from a small surrounding of $\Omega$.

Motion along the manifold $\Omega$ is induced by a projection of the vector field onto the tangent bundle of the manifold $\Omega$. If the manifold $\Omega$ is the quasi-equilibrium manifold for the macroscopic parameters $M$, then the motion along it is induced by the quasi-equilibrium projector

$$
P^{*} \bullet=\sum_{k} \frac{\partial f^{*}(M)}{\partial M_{k}}\left\langle\operatorname{Dm}_{k}(f) \mid \bullet\right\rangle \mid f^{*}(M) \cdot
$$

In the general case, if the manifold $\Omega$ is not quasi-equilibrium for the macroscopic variables $M$, the correct choice of the projector $P_{\Omega}$ for each state $f_{\Omega} \in \Omega$ is a non-trivial problem, and it is, in fact, well known from various 'closure problems'. For example, the Tamm-Mott-Smith approximation for strong shock waves [14] is based on the representation of the one-body distribution function in the form $\alpha f_{+}+(1-\alpha) f_{-}$, where $f_{ \pm}$are down- and upstream Maxwellians, and $\alpha$ is a parameter (the internal coordinate on the manifold). When a dynamic equation for $\alpha$ is derived from the Boltzmann equation, one needs to express $\alpha$ in terms of a moment (this is equivalent to choosing a projector). The difficulty here is that a priori there is no guide for how to choose this moment.

The problem of constructing the projector is solved in [12] on the basis of two considerations:

1. The vector field of the motions leading to the state $f_{\Omega} \in \Omega$ should be contained in the null-space of the projector $\operatorname{ker} P_{\Omega}$ : if the hypothesis about the decomposition of motions is correct, then the projection of rapid motions onto the manifold $\Omega$ should be small.

2. The entropy should increase in the course of rapid motions.

Therefore, if we accept that the hypothesis about the decomposition of motions is true, then each state $f_{\Omega} \in \Omega$ should be the maximum entropy state on the linear manifold of the form $f_{\Omega}+\operatorname{ker} P_{\Omega}$. If $\Omega$ is the quasi-equilibrium manifold, the set of planes $\operatorname{ker}_{\Omega}$ are parallel to each other for different individual states on this manifold (cf. Fig. 1), but if $\Omega$ is not quasi-equilibrium, then these planes are not parallel to each other any more. This construction of the projector for a manifold $\Omega$ implies that we do it as if the hypothesis of the decomposition of motions were true. This rule is used in the further steps of the method.

If the projector $P_{\Omega}$ is defined, one can write down the invariance equation:

$$
\left(1-P_{\Omega}\right) J\left(f_{\Omega}\right)=0 \text {. }
$$

The invariance equation (9) is solved iteratively. If the manifold $\Omega$ is not invariant (that is, the invariance condition (9) is not satisfied for each $f_{\Omega}$ ), we seek a correction $\delta f_{\Omega}$ such that

$$
\left\{\begin{array}{l}
P_{\Omega} \delta f_{\Omega}=0, \\
\left.\left(1-P_{\Omega}\right) D J\right|_{f_{\Omega}} \delta f_{\Omega}=-\left(1-P_{\Omega}\right) J\left(f_{\Omega}\right) .
\end{array}\right.
$$

Here, $\left.D J\right|_{f_{\Omega}}$ is the differential of the vector field $J$ evaluated in the state $f_{\Omega}$. Solving iteration (10), we obtain a set of new states $f_{\Omega}+\delta \Omega$ which build up a new (corrected) manifold $\Omega^{\prime}$.

Equations (10) are linear. Solutions to this equation build up a new manifold $\Omega^{\prime}$. Moreover, using the same idea of 'acting as if the decomposition of motions is true', we can replace the operator $D J$ with a self-adjoined operator. This new operator is taken self-adjoined with respect to the scalar product $\langle\mid\rangle_{S}$, defined by the quadratic approximation to the entropy:

$$
\langle g \mid h\rangle_{S}=-\left\langle g\left|D^{2} S\right| h\right\rangle,
$$


where $D^{2} S$ is the second differential of the entropy (represented, for instance, by the matrix of second-order derivatives). Construction of the self-adjoined operator makes use of the detail balance principle and Onsager relations as applied to rapid motions, and the explicit realization is given in [15].

Performing iterations along with the scheme

$$
\left(\Omega, P_{\Omega}\right) \rightarrow\left(\Omega^{\prime}, P_{\Omega}\right) \rightarrow\left(\Omega^{\prime}, P_{\Omega^{\prime}}\right),
$$

we obtain the sequence of manifolds $\Omega_{k}$ and projectors $P_{\Omega_{k}}$. In this sequence, each first step is the solution of the linearized invariance equation, while each second step is the construction of the new projector in accord with the rule given above. The use of an incomplete linearization should be stressed when going from Eq. (9) to (10), that is, derivatives of the projector $P_{\Omega}$ were not used. Besides purely technical reasons, this is motivated also by the fact that the iterations $\left(\Omega_{k}, P_{\Omega_{k}}\right)$ converge to the slowest invariant manifold (in the situations where this convergence can be proved), while the complete linearization converges only to the invariant manifold closest to the initial approximation.

It should be stressed that the method of invariant manifolds seeks true solutions of the original dynamics (or at least sufficient approximations thereof). In spite of the fact that the method is rather elaborative, it has been successful in a set of problems, in particular, the derivation of higher-order hydrodynamics from the Boltzmann and related equations [13,16,17], the construction of a reduced description for non-linear reaction kinetic equations $[15,18]$, and the establishment of the universal limit in the dilute polymer dynamics [19]. It is essential that the method of invariant manifold assumes dissipativity of the microscopic dynamics, and cannot be used to introduce dissipativity if it is absent from the input microscopic dynamics.

\subsection{Quasi-equilibria as approximations to invariant manifolds}

From the standpoint of the method of invariant manifold, the quasi-equilibrium manifolds are seen as relevant initial approximations. Already, the knowledge of the variance of the quasi-equilibrium approximation

$$
\Delta^{*}=\left(1-P^{*}\right) J\left(f^{*}\right)
$$

contains an information which helps to infer the form of the macroscopic equations of the first correction. Eq. (12) is simply the right-hand side of iteration (10) evaluated in the quasi-equilibrium approximation.

Let us look at an example. Let the microscopic variable $f(\boldsymbol{r}, \boldsymbol{v}, t)$ be the one-particle distribution function, obeying the Boltzmann kinetic equation $\dot{f}=-v_{\alpha} \partial_{\alpha} f+Q(f)$, where $Q$ is the Boltzmann collision integral. Subject to appropriate boundary conditions which we assume, this equation has the entropy $S=-k_{\mathrm{B}} \int f \ln f \mathrm{~d} \boldsymbol{v} \mathrm{d} \boldsymbol{r}$. The local Maxwell distribution function is the quasi-equilibrium approximation, the maximizer of the Boltzmann entropy subject to constraints $M=\left\{M_{0}, M_{\alpha}, M_{4}\right\}=\int\left\{1, v_{\alpha}, v^{2}\right\} f \mathrm{~d} \boldsymbol{v}$, where $\alpha=1, \ldots, d$, and where $d$ is the dimension of the microscopic velocity space. The macroscopic parameters $M$ are related to the usual local hydrodynamic fields, the mass, the momentum, and the energy density. The quasi-equilibrium dynamics is given by the Euler equations of inviscid fluid. Variance (12) is

$$
\Delta^{*}=-f^{*}\left\{\left[c^{2}-\frac{5}{2}\right]\left(v_{\alpha}-u_{\alpha}\right) T^{-1} \partial_{\alpha} T+2\left(c_{\alpha} c_{\beta}-\left(\frac{1}{3}\right) \delta_{\alpha \beta}\right) \partial_{\alpha} u_{\beta}\right\},
$$

where $\boldsymbol{c}=v_{\mathrm{T}}^{-1}(\boldsymbol{v}-\boldsymbol{u})$ is the peculiar velocity divided by the thermal velocity $v_{\mathrm{T}}=\left(2 k_{\mathrm{B}} T / m\right)^{1 / 2}, \boldsymbol{u}$ is the mean velocity, and $T$ is the kinetic temperature. It is clearly seen that the correction is due to the gradients 


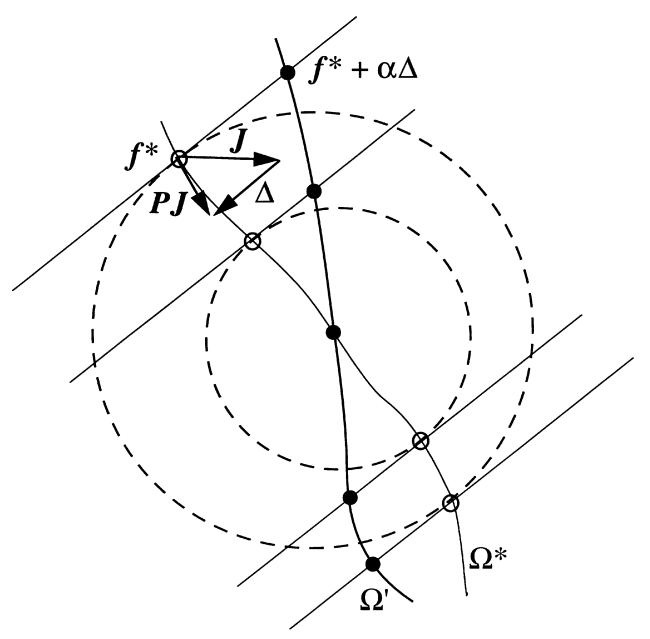

Fig. 2. The method of invariant manifold within the ansatz (14): the phase space and the quasi-equilibrium manifold are the same as in the Fig. 1. Non-invariance of the quasi-equilibrium approximation (open circles) is shown with the vector diagram. The correction (filled circles) is a shift of open circles in the direction of variance $\Delta$. Corrections form a new manifold $\Omega^{\prime}$ (bold line).

of the temperature and average velocity. When one solves the first iteration of the invariance equation (10) under the additional (simplifying) assumption that the characteristic time of collisions is much shorter than the characteristic time of free flight, the result is the Navier-Stokes equation. However, we can infer this result simply upon inspection of the variance. Indeed, let us consider the following ansatz:

$$
f^{\prime}=f^{*}+\alpha \Delta^{*}
$$

where $\alpha$ has the dimension of time. The form (14) satisfies the additional condition in Eq. (10), and can be used as a simple ansatz for solving this equation (which, in fact, amounts to an evaluation of the parameter $\alpha$ ). Under the same assumption about collision dominance, this simple ansatz leads again to the Navier-Stokes equations with values of transport coefficients estimated in the first-order Sonine polynomial approximation. This result is equivalent to the classical Chapman-Enskog solution under the same assumptions. Entirely the same considerations as applied to another well-known quasi-equilibrium, the Grad moment approximation, result in so-called Guyer-Krumhansl equations (see [20]).

The simple ansatz (14) is a guide for writing corrections to quasi-equilibrium approximations from the dissipative microscopic dynamics on semi-phenomenological grounds. The approximate solution to the iterations of the invariance equation via the ansatz (14) is sketched in Fig. 2.

\subsection{Invariance principle for hybridization of exact and approximate integration schemes}

A well-known problem of the non-Newtonian fluids is the problem of establishing constitutive equations on the basis of microscopic kinetic equations. We here consider a toy model introduced by Lielens et al. [21]:

$$
\dot{f}(q, t)=-\partial_{q}\left\{\kappa(t) q f-\frac{1}{2} f \partial_{q} U\left(q^{2}\right)\right\}+\frac{1}{2} \partial_{q}^{2} f .
$$


With the potential $U(x)=-(b / 2) \ln ((1-x) / b)$, Eq. (15) becomes the one-dimensional version of the well-known FENE dumbbell model. The reduced description seeks a closed equation for the stress $\tau=\left\langle q \partial_{q} U\right\rangle-1$. In this section, angular brackets denote averaging with $f$.

The kinetic equation (15) can be rewritten equivalently in terms of moment equations for the moments $M_{k}=\left\langle q^{2 k}\right\rangle$. Because of the non-polynomial character of the FENE potential, this moment chain has an infinite coupling: in each moment equation $\dot{M}_{k}=F_{k}$, the function $F_{k}$ depends on all moments.

We want to write the invariance equation in terms of moments. This is difficult for the FENE potential just because of the infinite-order coupling in the moment equations. In order to circumvent this difficulty, we first approximate the FENE equation with a hierarchy of kinetic equations for the potentials $U_{n}$, $n=0,1, \ldots$, formally converging to the FENE potential as $n$ tends to infinity.

The first two potentials are

$$
\begin{aligned}
& U_{0}=U^{\prime}\left(M_{1}\right) q^{2}, \\
& U_{1}=U_{0}+\frac{1}{2}\left(q^{4}-2 M_{1} q^{2}\right) U^{\prime \prime}\left(M_{1}\right)+\frac{1}{2}\left(M_{2}-M_{1}^{2}\right) q^{2} U^{\prime \prime \prime}\left(M_{1}\right),
\end{aligned}
$$

where $U^{\prime}, U^{\prime \prime}$ and $U^{\prime \prime \prime}$ denote the first, second and third derivative of the potential $U$, respectively. (The construction is described in [22], and here we indicate only the main ideas: first, we rewrite the force term on the right-hand side of Eq. (15) as $-(1 / 2) \partial_{q} f \partial_{q}\left(\delta S^{U} / \delta f\right)$, where $S^{U}=-\int U f \mathrm{~d} q$ is the spring contribution to the entropy. Next, after expanding the function $U$ into the power series in $q^{2}$, interchanging summation and integration, we come to a correlation function expansion of the functional $S^{U}$. Partial sums of this expansion are functionals $S_{0}^{U}, S_{1}^{U}$ etc., functional derivatives of which are the effective potentials $U_{0}, U_{1}$ etc.) The potentials $U_{n}$ are polynomials in $q^{2}$ with coefficients depending on the moments $M_{k}$ to the order $n+1$. Corresponding moment equations are therefore only finitely coupled in contrast to the full FENE potential. The potential $U_{0}$ corresponds to the well-known FENE-P model. The kinetic equation (15) with the potential $U_{1}(17)$ will be termed the FENE-P +1 model below. Direct Brownian dynamics (BD) simulation of the kinetic equation (15) with the potential $U_{1}$ for the flow situations studied in [21] demonstrates that it is a reasonable approximation to the true FENE dynamics, whereas the corresponding moment chain has a simpler structure (see Fig. 3).

For any potential $U_{n}$, the invariance equation can be studied directly in terms of the moments. Let us first consider the familiar FENE-P potential $U_{0}(16)$, where the moment chain has the following structure:

$$
\begin{aligned}
& \dot{M}_{k}=F_{k}\left(M_{k}, M_{k-1}, M_{1}\right), \\
& F_{k}=k(2 k-1) M_{k-1}+2 k\left[\kappa(t)-U^{\prime}\left(M_{1}\right)\right] M_{k} .
\end{aligned}
$$

We seek functions $M_{k}\left(M_{1}\right), k=2, \ldots$ which are form-invariant under the dynamics (18):

$$
\frac{\mathrm{d} M_{k}\left(M_{1}\right)}{\mathrm{d} M_{1}} F_{1}\left(M_{1}\right)-F_{k}\left(M_{k}\left(M_{1}\right), M_{k-1}\left(M_{1}\right), M_{1}\right)=0 .
$$

This set of invariance equations states the following: the time derivative of the form $M_{k}\left(M_{1}\right)$ when computed due to the closed equation for $M_{1}$ (the first contribution on the left-hand side of Eq. (19), or the 'macroscopic' time derivative) equals the time derivative of $M_{k}$ as computed by a true moment equation with the same form $M_{k}\left(M_{1}\right)$ (the second contribution, or the 'microscopic' time derivative), and this equality should hold whatsoever the values of the moment $M_{1}$ are.

Equations (19) are solvable exactly with the result

$$
M_{k}=a_{k} M_{1}^{k}, \quad \text { with } a_{k}=(2 k-1) a_{k-1}, a_{0}=1 .
$$




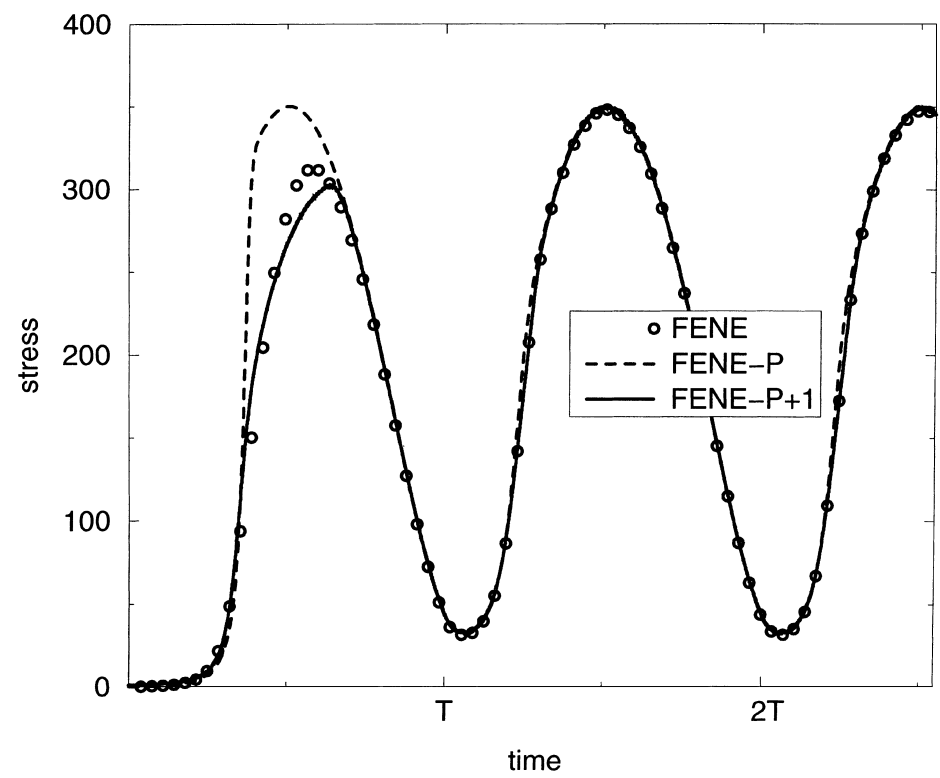

Fig. 3. Stress tensor vs. time from direct Brownian dynamics simulation for the periodic flow: symbols - FENE, dashed line - FENE-P (16), solid line - potential $U_{1}(17)$.

This dependence corresponds to the Gaussian solution in terms of the distribution functions. As expected, the invariance principle gives just the same result as the usual method of solving the FENE-P model.

In general, for the potential $U_{n}$, we seek functions of the form $M_{k}\left(M_{1}, M_{2}, \ldots, M_{n}\right), k=n+1, \ldots$ The invariance equations are written in the same pattern as for the FENE-P case just considered. Let us discuss the potential $U_{1}$, considering a closure approximation

$$
M_{k}=a_{k} M_{1}^{k}+b_{k} M_{2} M_{1}^{k-2},
$$

where $a_{k}=1-k(k-1) / 2$ and $b_{k}=k(k-1) / 2$. The ansatz (20) corresponds to the approximation used when deriving the potential $U_{1}$ (17). The function $M_{3}$ closes the moment equations for the two independent moments $M_{1}$ and $M_{2}$. The variance of this approximation is a set of functions $\Delta_{k}$ :

$$
\Delta_{k}\left(M_{1}, M_{2}\right)=\frac{\partial M_{k}}{\partial M_{1}} F_{1}+\frac{\partial M_{k}}{\partial M_{2}} F_{2}-F_{k},
$$

In the sequel, we make all conclusions based on the variance $\Delta_{3}(21)$. It is instructive to plot the variance $\Delta_{3}$ versus time, assuming the functions $M_{1}$ and $M_{2}$ are found by the BD simulation (see Fig. 4). We observe that the variance is a non-monotonic function of time, and that there are three pronounced domains: from $t_{0}=0$ to $t_{*}$ the variance is almost 0 , which means that the ansatz is reasonable. In the intermediate domain, the variance jumps to high values (so the quality of approximation is poor). However, after some time $t=t^{*}$, the variance again becomes negligible, and remains so for later times in spite of the fact that the flow is periodic. Such a behavior is typical of the so-called 'kinetic layer'. The BD simulation for the distribution function helps to understand what happens between the times $t_{*}$ and $t^{*}$ : the shape of the distribution function changes rapidly from Gaussian-like at $t_{*}$ to almost a delta-functional form at $t^{*}$, and the latter form does not change qualitatively at later times (similar behavior is found for the FENE 


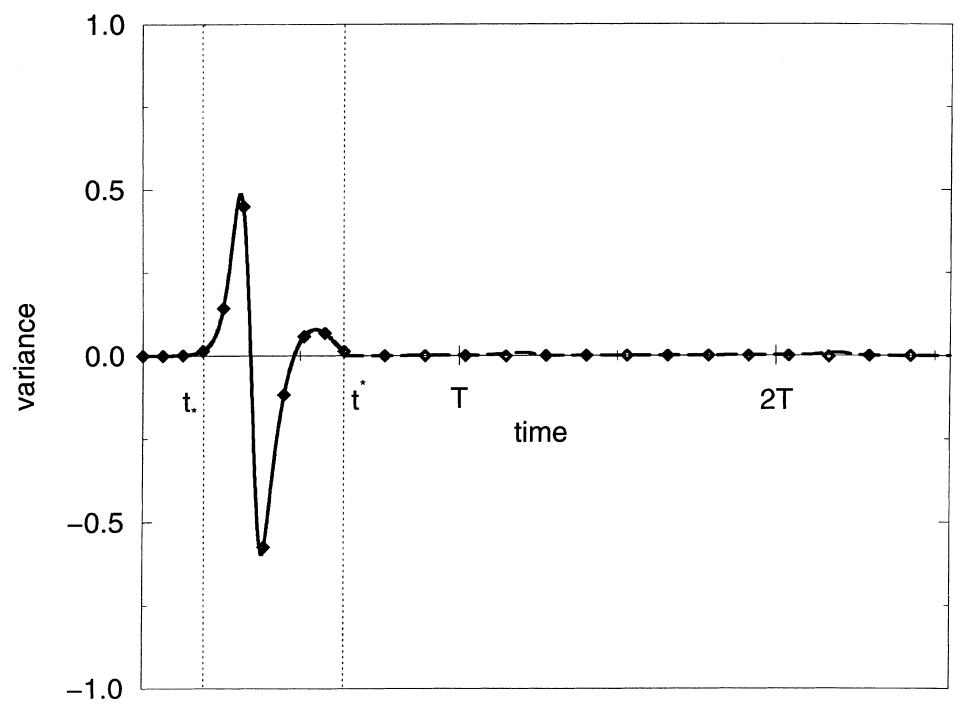

Fig. 4. The variance $\Delta_{3}$ of the closure (20): symbols and the solid line - the time dependence of the moments $M_{1}$ and $M_{2}$ was taken from the BD simulation, dashed line - the time dependence of the moments $M_{1}$ and $M_{2}$ was taken from the closed macroscopic equation. The BD data for times after $t^{*}$ is given for comparison.

model also [21]). It should be stressed that it is the closure (20) that 'sees' the domain between $t_{*}$ and $t^{*}$ as the kinetic layer, and not the kinetic model itself. In other words, 'good' closures will see less kinetic layers than 'poor' closures (the limiting case is the invariant closure which sees no kinetic layers at all, as it is in the case of the FENE-P model). Similar considerations are valid elsewhere: for instance, it is the Navier-Stokes (macroscopic) equation which tends not to follow the solution of the Boltzmann equation at high Mach number shock layers.

Instead of attempting to improve the closure analytically, the invariance principle can be used directly to switch from the BD simulation to the solution of the macroscopic equation without losing the accuracy to a given tolerance. Indeed, the variance is a function of $M_{1}$ and $M_{2}$, and it can be easily evaluated both on the data from the solution to the macroscopic equation, and the BD data. If the variance exceeds some given tolerance on the macroscopic solution, this signals to switch to the BD integration. On the other hand, if the variance becomes less than the tolerance level on the $\mathrm{BD}$ data, it is a signal that the BD simulation is not necessary anymore, and one can continue with the integration of the macroscopic equations. This reduces the necessity of using BD simulations only to get through the kinetic layers. A realization of this hybrid approach is demonstrated in Figs. 5 and 6: for the same flow, we have used the BD dynamics only from $t=0$ to $t=t^{*}$, while we have integrated the macroscopic equations at all later times. The quality of the result is comparable to the BD simulation, whereas the total integration time is much shorter. The transient dynamics at the point of switching from the BD scheme to the integration of the macroscopic equations deserves a special comment: the initial conditions at $t^{*}$ are taken from the BD data. Therefore, we cannot expect that at the time $t^{*}$ the solution is already on the invariant manifold, rather, it is at best, close to it. Transient dynamics therefore signals the stability of the invariant manifold we expect: even though the macroscopic solution does not start on this manifold, it nevertheless is attracted to it. The transient dynamics becomes progressively less pronounced if the switching is done at later times. 


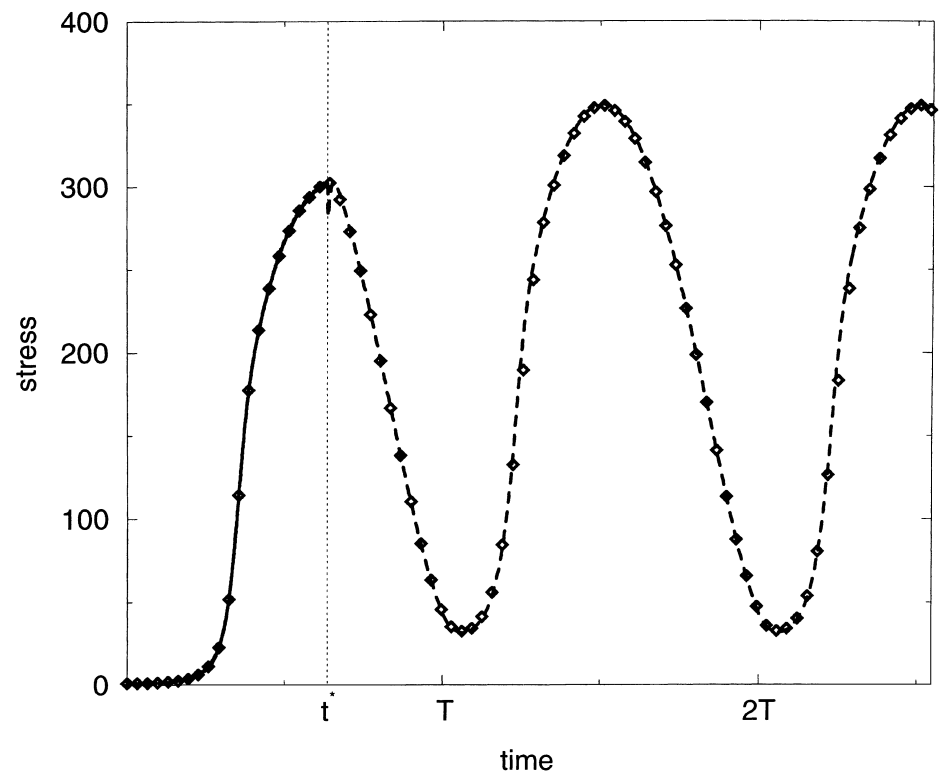

Fig. 5. Switching from the BD simulation to the macroscopic equation after the variance has reached the given tolerance level: symbols — the BD simulation, solid line — the BD simulation from $t=0$ to $t=t^{*}$, dashed line — integration of the macroscopic dynamics with the initial data from the BD simulation at $t=t^{*}$. The point of switching is characterized by a short period of transient dynamics.

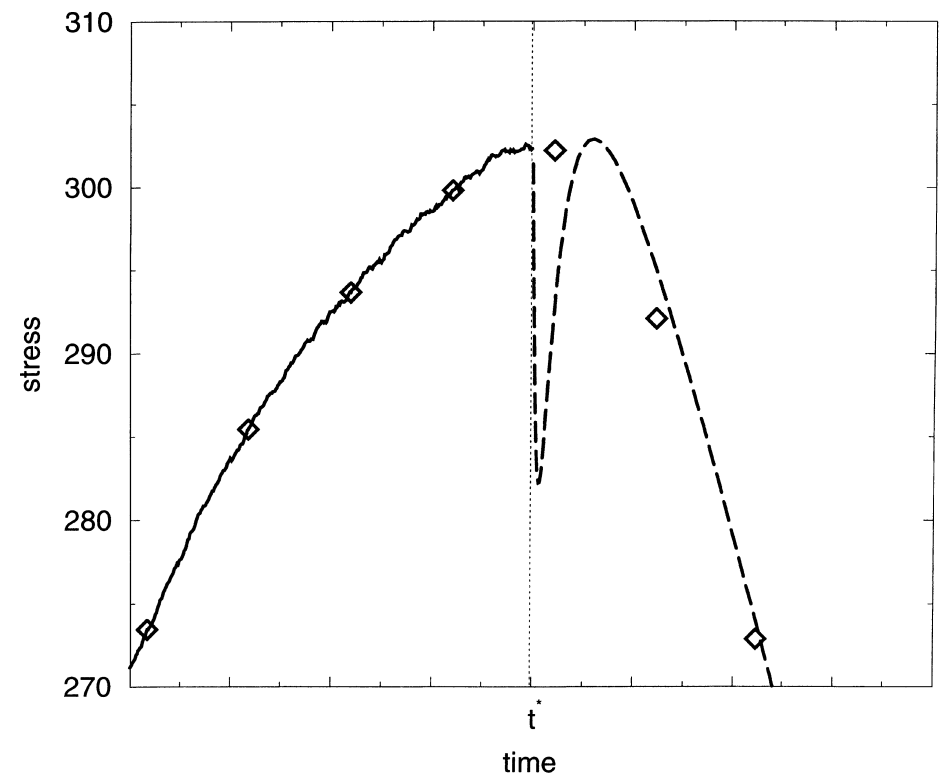

Fig. 6. Transient dynamics at the switching from BD to macroscopic equation on a finer time scale. 
This approach can be realized on the level of moment closures (which then needs reconstruction of the distribution function from the moments at the switching from macroscopic integration to BD procedures), or for parametric sets of distribution functions if they are available [21].

\section{Enhancement of quasi-equilibrium approximations for entropy-conserving dynamics}

\subsection{Entropy-conserving dynamics with periodic coarse-graining}

On the 'most microscopic' level, the level of individual particles, dynamics conserves the entropy. Thus, as we have already mentioned in Section 1, neither the quasi-equilibria nor any of its corrections (in the sense of Section 2) can bring in the dissipativity. Nevertheless, the quasi-equilibrium approximations play the central role in deriving dissipative dynamics from the entropy-conserving dynamics. Actual implementations of this derivation may differ from one another. It is the goal of the present section to describe the simplest analytic implementation, the microscopic motion with periodic coarse-graining. The notion of coarse-graining was introduced by P. Ehrenfest and T. Ehrenfest in their seminal work [10]: the phase space is partitioned into cells, and the coarse-grained variables are the amounts of the phase density inside the cells. Dynamics is described by two processes, by the Liouville equation for $f$, and by periodic coarse-graining, replacement of $f(x)$ in each cell by its average value in this cell. The coarse-graining operation means forgetting the microscopic details, or the history.

From the perspective of general quasi-equilibrium approximations, periodic coarse-graining amounts to the return of the true trajectory on the quasi-equilibrium manifold with the preservation of macroscopic variables. The motion starts at the quasi-equilibrium state $f_{i}^{*}$. Then, the true solution $f_{i}(t)$ of the microscopic equation (2) with the initial condition $f_{i}(0)=f_{i}^{*}$ is coarse-grained at a fixed time $t=h-$ the function $f_{i}(h)$ is replaced by the quasi-equilibrium function $f_{i+1}^{*}=f^{*}\left(m\left(f_{i}(h)\right)\right)$. The entropy-conserving dynamics with periodic coarse-graining is visualized in Fig. 7. From the features of the quasi-equilibrium approximation, we have the following:

Proposition. For the motion with periodic coarse-graining, the following inequality is valid:

$$
S\left(f_{i}^{*}\right) \leq S\left(f_{i+1}^{*}\right)
$$

while the equality occurs if and only if the dynamic system (2) conserves the entropy, and the segment of the true trajectory $f_{i}(t)$ between $t=0$ and $t=h$ belongs to the quasi-equilibrium manifold.

Thus, if this process of motion with periodic coarse-graining is continued, and if the quasi-equilibrium is not the invariant manifold of the microscopic dynamics, the values $S\left(f_{i}^{*}\right)$ form a monotonically increasing sequence. It is important to stress here that the quasi-equilibrium manifold used in the construction must not be the invariant manifold of the microscopic dynamics: if the true trajectory cannot 'take off' from the manifold, the entropy remains constant all the time.

\subsection{Second-order dissipative dynamics and the entropy production formula}

The actual implementation of the periodically coarse-grained motion still requires integration of the Liouville equation for time $h$. This certainly can be done only formally. In this section, we consider 


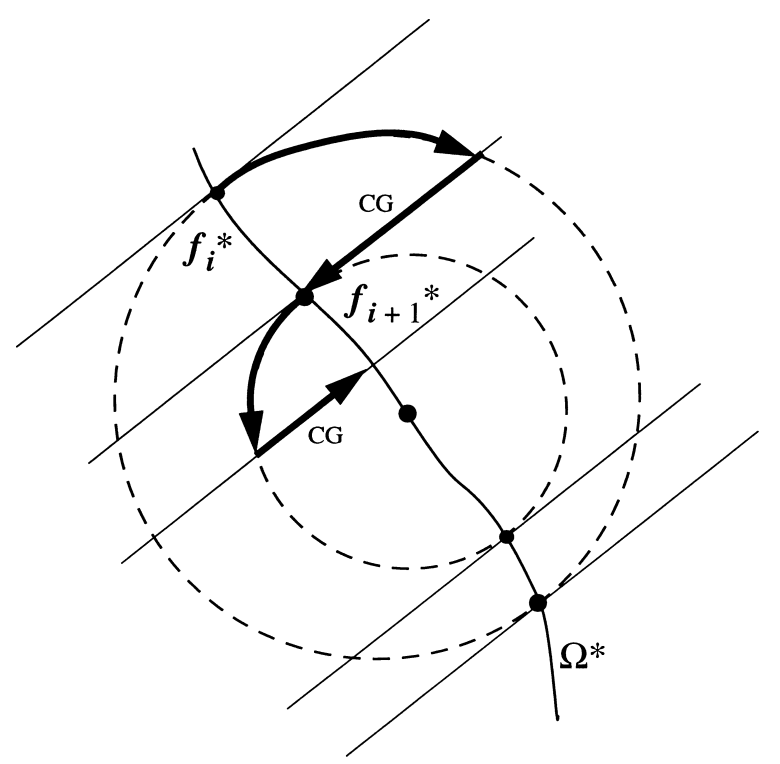

Fig. 7. Entropy-conserving dynamics with periodic coarse-graining: curved arrows represent the microscopic solutions, and straight arrows represent the coarse-graining.

an approximate implementation, dealing with second-order accurate solutions to the Liouville equation rather than with full solutions.

We consider linear macroscopic variables, and write $M_{k}(f)=\left\langle m_{k} \mid f\right\rangle$, assuming linear independence $\left(\left\langle m_{k} \mid \partial f^{*} / \partial M_{j}\right\rangle=\delta_{k j}\right)$. Let us write down the solution to the microscopic equation (2) to second order in $h$, subject to the quasi-equilibrium initial condition:

$$
f(h)=f^{*}+h J^{*}+\frac{h^{2}}{2} D J^{*}\left|J^{*}\right\rangle+\mathrm{o}\left(h^{2}\right) .
$$

Here, $D J^{*}$ is the first differential of the vector field $J$ evaluated in the quasi-equilibrium. This scheme is entropy-conserving, that is $S(h) \equiv S(f(h))=S^{*}(M)+\mathrm{o}\left(h^{2}\right)$. Evaluation of the macroscopic variables on the function (23) gives

$$
M_{k}(h)=M_{k}+h \varphi_{k}^{*}(M)+\frac{h^{2}}{2}\left\langle m_{k}\left|D J^{*}\right| J^{*}\right\rangle+\mathrm{o}\left(h^{2}\right),
$$

where $\varphi_{k}^{*}(M)=\left\langle m_{k} \mid J^{*}\right\rangle$ is the quasi-equilibrium macroscopic vector field, the right-hand side of Eq. (3).

We shall now establish the macroscopic dynamic equations by matching the macroscopic and the microscopic dynamics at the collocation points. Specifically, the macroscopic dynamic equations, $\dot{M}_{k}=\varphi_{k}(M)$ with the right-hand side not yet defined, give the following second-order result:

$$
M_{k}(h)=M_{k}+h \varphi_{k}+\frac{h^{2}}{2} \sum_{j} \frac{\partial \varphi_{k}}{\partial M_{j}} \varphi_{j}+\mathrm{o}\left(h^{2}\right) .
$$


Assuming the functions $\varphi_{k}$ in the form $\varphi_{k}=\varphi_{k}^{*}+h R_{k}$, and requiring the matching of Eqs. (24) and (25) to the order $h^{2}$ for every coarse-graining event, we find

$$
\begin{aligned}
& \dot{M}_{k}=\varphi_{k}^{*}+h R_{k}, \\
& R_{k}=\frac{1}{2}\left[\left\langle m_{k}\left|D J^{*}\right| J^{*}\right\rangle-\sum_{j} \frac{\partial \varphi_{k}^{*}}{\partial M_{j}} \varphi_{j}^{*}\right]=\frac{h}{2}\left\langle m_{k}\left|D J^{*}\right|\left(1-P^{*}\right) J^{*}\right\rangle,
\end{aligned}
$$

where $P^{*}$ is the quasi-equilibrium projector (8). Macroscopic equations (26) enhance the quasi-equilibrium dynamics, and unlike the latter, they are dissipative. The use of the fixed period at which the coarse-graining is done amounts to a short memory assumption.

Why the macroscopic entropy production is expected to be positive in the dynamics (26) follows from the proposition mentioned above: by our assumption, the quasi-equilibrium is not the solution to the dynamic equations (2); therefore, any true solution when started at the quasi-equilibrium manifold does not belong as a whole to this manifold. The true value of the entropy at time $h$ is therefore less than the value of the quasi-equilibrium entropy $S^{*}$ computed from the corresponding values of the macroscopic variables. Coarse-graining releases this entropy difference, and because the scheme (23) conserves the entropy, this consideration remains valid to the relevant (second) order.

A direct computation demonstrates that the entropy production due to the dynamics (26) has the following explicit form:

$$
\dot{S}^{*}=-\frac{h}{2}\left\langle\left(1-P^{*}\right) J^{*}\left|D^{2} S^{*}\right|\left(1-P^{*}\right) J^{*}\right\rangle=-\frac{h}{2}\left\langle\Delta^{*} \mid \Delta^{*}\right\rangle_{S},
$$

where $\Delta^{*}$ is the variance of the quasi-equilibrium approximation, familiar from Section 2 . The right-hand side of Eq. (27) is a non-negative definite form because the entropy functional $S$ is concave, and it equals 0 only if the quasi-equilibrium approximation is the true solution to the originating dynamics, in agreement with the remarks given above.

In order to illustrate the results (26) and (27), we shall consider again the case of the one-particle distribution function, while the entropy-conserving dynamics is given by the streaming operator (that is, we consider again the 'Boltzmann equation' as in the Section 2 but this time without the collision operator). Then, the quasi-equilibrium dynamics is again the Euler (inviscid) dynamics, the variance of the local Maxwell manifold is again given by Eq. (13), and the terms (26c) are the Navier-Stokes-Fourier dissipation terms with transport coefficient proportional to $h$. Thus, the periodic coarse-graining procedure in this example replaces the collisions, and approximates the transport coefficients essentially in the same way as it does the ansatz (14), that is, in this example, the physically meaningful interpretation of the time $h$ between subsequent coarse-grainings is the characteristic free flight time. Several comments are in order:

1. The periodically coarse-grained motion can be seen as a motion with fluctuations around the quasiequilibrium manifold. The 'driving force' of the fluctuations is the variance $\Delta^{*}=\left(1-P^{*}\right) J^{*}$. It is therefore not surprising that the form of the entropy production (27) is similar to the standard expression of the fluctuation-dissipation theorem, where the time integral is approximated by the time $h$.

2. In view of the previous remark, it should be stressed that, within our approach, the kinetic coefficients in the resulting macroscopic equations are only crude approximations when compared to standard 
expressions containing the memory integral. In particular, all transport coefficients are proportional to one characteristic time scale $h$. Nevertheless, the real advantage of the approach is its simplicity. This is crucial when relevant quasi-equilibria are of a complicated form (such as, for example, the quasi-equilibrium for the pair correlation function as the macroscopic variable [23]). Analysis of quasi-equilibrium approximations for 'non-standard' macroscopic variables is, with no doubt, one of the important steps in the development of the theory of complex fluids. Derivation of macroscopic equations presented here is advantageous when the form of the resulting dissipative equations is difficult to guess.

Furthermore, the procedure of periodic coarse-graining can be extended in order to take into account the (possible) memory effects and spectra of relaxation times. There are at least two natural ways for such an extension: first, the order of approximation of the trajectory can be extended from the second to a higher order. Second, instead of just one coarse-graining per time step, one can take the averaged state after several coarse-grainings over a larger time segment. It can be demonstrated that, in the limit of the infinite time segment, the result formally converges to Zubarev's method of non-equilibrium statistical operator [1-8]. A detailed study of these questions is the matter of a separate investigation.

\section{Final remarks}

In this paper, we have discussed the two main routes along which the quasi-equilibrium states enter the non-equilibrium thermodynamics. The focus of our discussion in both cases (corrections and enhancement) was: once we have a quasi-equilibrium manifold, can we do something 'better' out of it? We therefore have not focused on a more traditional use of quasi-equilibrium ensembles 'as is'. Finally, a further progress can be expected when linking the results of corrections and enhancement of quasi-equilibrium ensembles with the recent advances in writing macroscopic equations following certain patterns or structural prescriptions. Some of these prescriptions are discussed in several contributions to the present collection of papers.

\section{References}

[1] G.W. Gibbs, Elementary Principles of Statistical Mechanics, Dover, New York, 1960.

[2] E.T. Janes, Papers on probability, statistics and Statistical physics, ed. R.D. Rosenkrantz, Creidel, Dordrecht, 1983.

[3] D. Zubarev, V. Morozov, G. Röpke, Statistical Mechanics of Nonequilibrium Processes, Vol. 1, Basic Concepts, Kinetic Theory, Akademie Verlag, Berlin, 1996, Vol. 2, Relaxation and Hydrodynamic Processes, Akademie Verlag, Berlin, 1997.

[4] H. Grad, On the kinetic theory of rarefied gases, Comm. Pure Appl. Math. 2 (4) (1949) 331-407.

[5] A.M. Kogan, L.I. Rozonoer, On the macroscopic description of kinetic processes, Dokl. ANSSSR 158 (3) (1964) $566-569$.

[6] A.M. Kogan, Derivation of Grad-type equations and study of their properties by the method of entropy maximization, Prikl. Math. Mech. 29 (1) (1965) 122-133.

[7] L.I. Rozonoer, Thermodynamics of nonequilibrium processes far from equilibrium, in: Thermodynamics and Kinetics of Biological Processes, Nauka, Moscow, 1980, pp. 169-176.

[8] A.N. Gorban, Equilibrium Encircling, Chapter 3, Quasi-equilibrium and entropy maximum, Nauka, Novosibirsk, 1984, pp. 92-122.

[9] D. Jou, J. Casas-Vázquez, G. Lebon, Extended Irreversible Thermodynamics, Springer, Berlin, 1993.

[10] P. Ehrenfest, T. Ehrenfest, Begriffliche Grundlagen der statistischen Auffassung in der Mechanik, Enzyklopüdie der Mathemathischen Wissenschaften, Bd. IV 2, II, Heft 6, 1911. P. Ehrenfest, Collected Scientific Papers, North-Holland, Amsterdam, 1959, pp. 213-300 (reprint). 
[11] C. Tsallis, Possible generalization of Boltzmann-Gibbs statistics, J. Stat. Phys. 52 (1/2) (1988) 479-487.

[12] A.N. Gorban, I.V. Karlin, Thermodynamic parameterization, Physica A 190 (1992) 393-404.

[13] A.N. Gorban, I.V. Karlin, Method of invariant manifolds and regularisation of acoustic spectra, Transport Theory Stat. Phys. 23 (1994) 559-632.

[14] C. Cercignani, Theory and Application of the Boltzmann Equation, Scottish Academic Press, Edinburgh, 1975.

[15] A.N. Gorban, I.V. Karlin, V.B. Zmievskii, S.V. Dymova, Reduced description in reaction kinetics, Physica A 275 (2000) 361-379.

[16] A.N. Gorban, I.V. Karlin, Short-wave limit of hydrodynamics: a soluble example, Phys. Rev. Lett. 77 (1996) $282-285$.

[17] I.V. Karlin, G. Dukek, T.E. Nonnenmacher, Invariance principle for extension of hydrodynamics: nonlinear viscosity, Phys. Rev. E 55 (2) (1997) 1573-1576.

[18] V.I. Bykov, A.N. Gorban, I.V. Karlin, S.V. Dymova, V.B. Zmievskii, Method of invariant manifolds for the reduction of kinetic description, in: G.D. Roy, S.M. Frolov, P. Givi (Eds.), Advanced Computation and Analysis of Combustion, ENAS Publishers, Moscow, 1997, pp. 14-24.

[19] V.B. Zmievskii, I.V. Karlin, M. Deville, The universal limit in dynamics of dilute polymeric solutions, Physica A 275 (2000) $152-177$.

[20] I.V. Karlin, A.N. Gorban, G. Dukek, T.F. Nonnenmacher, Dynamic correction to moment approximations, Phys. Rev. E 57 (1998) 1668-1672.

[21] G. Lielens, P. Halin, I. Jaumain, R. Keunings, V. Legat, New closure approximations for the kinetic theory of finitely extensible dumbbells, J. Non-Newtonian Fluid Mech. 76 (1998) 249-279.

[22] P. Ilg, I.V. Karlin, H.C. Öttinger, Generating moment equations in the Doi model of liquid-crystalline polymers, Phys. Rev. E 60 (1999) 5783-5787.

[23] N.N. Bugaenko, A.N. Gorban, I.V. Karlin, Universal expansion of the triplet distribution function, Teoreticheskaya i Matematicheskaya Fizika 88 (1991) 430-441 (Trans.; Theoret. Math. Phys. (1992) 977-985). 